\title{
Capital Markets and Foreign Ownership Restrictions: An Empirical Analysis of Emerging Stock Markets
}

\author{
JAVED ANWAR and M. TARIQ JAVED
}

\section{INTRODUCTION}

In the 1990s, the hot issue in international finance was the growing interest of portfolio managers in the emerging stock markets. The interest in the emerging markets gained rapid attention, which is evident from the global trends, towards the opening up of economies and financial markets, free capital flow and the privatisation of financial institutions. Earlier the emerging markets were isolated due to several factors that had posed serious problems for international investors. These markets lacked the depth, regulatory framework, and structural safeguards that had characterised the equity markets in the developed world.

Capital markets are called integrated, if assets with perfectly correlated rates of returns have the same price regardless of the location in which they are traded. Alternatively, capital market are called segmented, if financial assets traded in different markets "with identical risk characteristics" have different returns due to different investment restrictions. ${ }^{1}$ Segmentation may be due to individuals' attitudes, government restrictions over capital movements or irrationality.

In the past twenty-five years, modern finance theory has proved to be a major development in finance, which comprises of portfolio theory, capital market theory and efficient market theory. These modern developments can be traced back to the work of Markowitz (1959); Sharpe (1964); Solnik (1974) etc., which assumes that security prices fully reflect all publicly available information. Due to this information, potential investors can gain benefits through international diversification. The major attraction of forming international portfolios lies in the potential for risk reduction through diversification of unsystematic risk. The lower the correlation among the asset returns internationally, the higher is the reduction in risk. This insight has led to the development of well-known Capital Asset Pricing Model

Javed Anwar is Teaching Fellow at Mohammad Ali Jinnah University, Islamabad. M. Tariq Javed is Assistant Professor, Department of Economics, Quaid-i-Azam University, Islamabad.

${ }^{1}$ Various types of restrictions to international investment are briefly discussed in Section II. 
(CAPM). Emerging markets returns have low levels of correlation thus reflecting low levels of economic integration. The return correlation depends on the degree of capital market integration and reflects spill over effect across international markets. ${ }^{2}$

In this study a general asset-pricing model has been used to compare the integration of emerging stock markets with the global capital market. Five forecasting variables including two domestic instruments (emerging market lagged returns and dividend yield) and three global instruments (U.S. lagged returns, ${ }^{3}$ dividend yield and interest rate) have been used to test the hypothesis. Previously, Bekaert (1995); Campbell and Hamao (1992) have tried similar variables for capital market integration. Similarly, Bekaert and Hodrick (1992) have also used dividend yield to predict excess returns in the industrial equity markets. Campbell and Ammer (1993) have also used dividend yield as a proxy for the long-horizon expected excess returns.

The main emphasis of Campbell and Hamao (1992) was on two countries i.e. Japan and U.S., while Bekaert (1995) has used comparatively smaller sized sample. Our work is different in a sense that we are looking for the foreign investment restrictions impact, although not directly, on the linear association of regression coefficients of the expected returns in emerging stock markets and the U.S. market. We expect that there will be differences between expected returns of emerging markets ${ }^{4}$ and the U.S. market across the two sample periods.

There are two major approaches to test and measure the degree of market segmentation/integration.

In the first approach we look for direct evidence of barriers to investment across markets i.e. legal restrictions on foreign share ownership, transaction taxes, exchange rate control etc., or of a limited scale of cross-border transactions in financial assets. ${ }^{5}$

The second approach assumes that markets are integrated and that some mean-variance efficient benchmark portfolio is observable. If this assumption holds, then assets traded in integrated capital markets have expected returns that are determined by their observable betas ${ }^{6}$ with the benchmark return and by the observable mean benchmark return. These betas, commonly, are assumed to be constant over time. However some recent works has started to allow variation in betas with certain conditioning variables. ${ }^{7}$

\footnotetext{
${ }^{2}$ See for detail Hamao et al. (1991).

${ }^{3}$ U.S. lagged returns are used as a proxy for the global market lagged returns.

${ }^{4}$ The term "emerging markets" can imply that a process of change is underway, with stock markets growing in size and sophistication, in contrast to markets - that are small and stagnant.

${ }^{5}$ French and Poterba (1990) study the extent to which U.S. and Japanese investors make crossborder investment in common stock.

${ }^{6}$ Betas mean responsiveness of stock market returns to its determinants.

${ }^{7}$ See Cho, Eun, and Senbet (1986); Gultekin, Gultekin and Penati (1989); Jorion and Schwartz (1986); Stehle (1977); Wheatley (1988) and Harvey (1991).
} 
The objective of the study is to investigate the linear association of regression estimates of the expected returns in emerging stock markets and the U.S. stock market. This linear association is an indicator of the common component in expected returns and hence an indirect measure of market segmentation. The analysis has been performed for two different sample periods.

- Pre-Financial Reforms period in emerging stock markets (Jan.1989- Dec. 1991).

- Post-Financial Reforms period in emerging stock markets (Jan.1992-Dec. 1998).

The study is spread over six sections and organised in such a way that Section II briefly discusses the investment barriers in emerging markets. Data and methodology are explained in Section III. Section IV provides a discussion of the empirical results of the study. Section $\mathrm{V}$ summarises the results of structural change and the last section gives a brief conclusion of the study.

Emerging Stock Markets are ideal for investment due to its high returns. Portfolio investment in these markets has increased in the mid-90s after the financial liberalisation. International investments had been restricted in the emerging stock markets up-till 1989 when financial and exchange rate restrictions had started to relax. These processes were continued slowly up-till 1992 when almost all restrictions to international investment were abolished. This investment barrier in emerging markets discouraged investments and lead to de facto segmentation. For the purpose of our research, we have given labels to the period of restrictions as Pre-financial Libralisation period and to the period after financial reforms as Post-financial Libralisation period. Different types of investment barriers in emerging markets are discussed below.

First are legal barriers arising from the different status of foreign and domestic investors. These are direct restrictions on foreign ownership. For example, certain sectors may be closed to foreign investment, or limits may be imposed on direct ownership of equity.

Second types of direct barriers are exchange and capital controls that affect investment in emerging markets. For example, some economies have direct restrictions, such as a minimum investment period, on the remittance of profits. Taxes on dividends and capital gains are considered direct barriers in this group.

Third are indirect barriers arising from differences in available information, accounting standards, and investor's protection. Investors might not have adequate information on these markets and on the financial condition of the companies, the settlement system might be inefficient and slow, accounting standards might be poor, and investor protection might be minimal. These factors might play a crucial role in the investment decisions of international investors. Chuhan (1992) has listed limited information on emerging markets as one of the key impediments to investment in emerging markets. 
Other indirect barriers are arising from emerging market specific risks (EMSRS). EMSRS includes liquidity risk, political risk, economic policy risk, macro-economic instability, and currency risk. Political instability and economic mismanagement may add substantial risk premium to returns and deter some foreign investors. A crude and indirect measure of political risk is the secondary market price of bank debt.

The second EMSRS is liquidity risk because liquidity may be correlated with the size of the stock market. Turnover measure (Value traded as a percentage of market capitalisation) can serve as a liquidity indicator.

These types of restrictions have tendency to pose some serious problems for the potential investors and lead to isolate the emerging stock markets from the global capital market.

\section{DATA AND METHODOLOGY}

For the analysis of capital market, we have selected a sample of nineteen emerging stock markets and USA stock market (used as a proxy for the world market). Since data for Pakistan are available from January 1989, therefore we picked those markets whose data set was similar to the data of Pakistan market. Indonesia, China and other emerging markets are excluded due this fact. The sample countries have been split into four groups as:

\begin{tabular}{ll}
\hline Group & Markets \\
\hline Latin America & Argentina, Brazil, Chile, Colombia, Mexico, \\
& Venezuela. \\
East Asia & Korea, Philippines, Taiwan(China). \\
South Asia & India, Malaysia, Pakistan, Thailand. \\
Europe/Mid-East/Africa & Greece, Jordan, Nigeria, Portugal, Turkey, \\
& Zimbabwe. \\
\hline
\end{tabular}

The analysis is based on monthly IFCG price indices. The data are taken from various issues of Emerging Stock Markets Fact Book, International Finance Corporation. Data on interest rate (T-Bill) of USA are collected from International Financial Statistics. All the price indices are given in U.S. dollar for a sample of 120 monthly observations (Jan.1989 to Dec.1998). These indices are calculated with the base year of Dec.1984, where as the base years for Portugal and Turkey is Jan.1986 and Dec.1986. So for an easy comparison all indices are converted to the common base of Jan.1989. Returns are calculated from the IFCG regional price indices. We will use two local instruments (local lagged returns and dividend yield) and three global instruments (U.S. lagged returns, U.S. dividend yield and U.S. interest rate) as forecasting variables in our regression analysis. For this purpose, we will use a general asset-pricing model. 
The most general asset-pricing model ${ }^{8}$ is a K-factor model of the following form:

$$
\tilde{r}_{i, t+1}=E_{t}\left[\tilde{r}_{i, t+1}\right]+\sum_{k=1}^{K} \beta_{i k} \tilde{f}_{k, t+1}+\tilde{\varepsilon}_{i, t+1} \quad \ldots \quad \ldots \quad \ldots \quad \ldots
$$

Here $r_{i, t+1}$ is the return on asset $i$ held from time $t$ to $t+1$. The return on asset $i$ equals the expected return, plus the sum of $K$-factor realisation $f_{k, t+1}$ times their betas or factor loading $\beta_{i k}$, plus an idiosyncratic error term $\varepsilon_{i, t+1}$. The asset-pricing model is dynamic in the sense that the expected return can vary through time, but static in that the beta coefficient are assumed to be constant through time.

The model restricts the expected return as follows:

$$
E_{t}\left[\tilde{r}_{i, t+1}\right]=\sum_{i=k}^{K} \beta_{i k} \lambda_{k t} \quad \ldots \quad \ldots \quad \ldots \quad \ldots \quad \ldots
$$

where $\lambda_{k t}$ is the market price of risk for the $k$ th factor at time $t$.

Now suppose that the information set at time $t$ consist of a vector of $N$ forecasting variables $X_{n}, n=1, \ldots ., N$ (where $X_{1 t}$ is a constant). The variables include U.S. lagged returns, emerging markets lagged return, emerging market and U.S. market dividend yields and U.S. interest rate (U.S. T-bill rate), and that conditional expectations are linear in these variables. Then the $k$ th risk price can be written

$$
\begin{array}{llllllll}
\lambda_{k t}=\sum_{n=1}^{N} \theta_{k n} X_{n t} & \ldots & \ldots & \ldots & \ldots & \ldots & \ldots
\end{array}
$$

and Equation (2) becomes

$$
E_{t}\left[\tilde{r}_{i, t+1}\right]=\sum_{k=1}^{K} \beta_{i k} \sum_{n=1}^{N} \theta_{k n} X_{n t}=\sum_{n=1}^{N} \alpha_{i n} X_{n t} \quad \ldots \quad \ldots \quad \ldots
$$

Equation (4) says that the $I N$ coefficient $\alpha_{i n}$ are obtained by regressing $I$ excess return on $N$ forecasting variables that can be written in terms of $K$ beta coefficients and $N$ theta coefficients which define market price of risk.

Suppose we observe a portfolio whose return has a beta of one on the first factor, and zero on the other factors. Suppose further that the return on this portfolio has zero idiosyncratic risk. Call the return on this portfolio $r_{i, t+1}$. Then we have

$$
\begin{aligned}
& \tilde{r}_{i, t+1}=\beta_{i 1} \tilde{r}_{1, t+1}+\sum_{k=2}^{K} \beta_{i k} \sum_{n=1}^{N} \theta_{k n} X_{n t}+\sum_{k=2}^{K} \beta_{i k} \tilde{f}_{k, t+1}+\tilde{\varepsilon}_{i, t+1}
\end{aligned}
$$

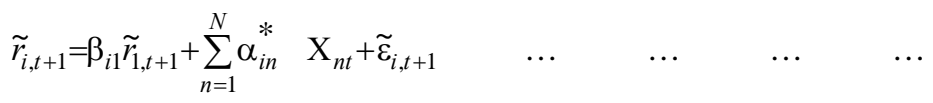

${ }^{8}$ This type of model has also used by Campbell and Hamao (1992); Bekaert (1995); Bekaert and Hodrick (1992) for capital market analysis. 
In this study we use forecasting variables $X_{n t}$ which are known to the market at time $t$. Generally, it is not assumed that all the relevant variables are included, but the method described above is robust to omitted information. By taking conditional expectations of Equation (5), it is straightforward to show that the various restrictions hold in the same form when a subset of the relevant information is used.

Thus if the coefficients $\alpha_{i n}^{*}$ in Equation (5) are zero for true information vector used by the market, they will also be zero if a subset of this vector is included in Equation (5). Similarly, if the market's forecasts of excess returns in the two countries are perfectly correlated, then forecasts using a subset of the market's information must also be perfectly correlated.

\section{EMPIRICAL FINDINGS}

Table 1 reports the results of pre-financial reforms period in which foreign investors have no access to emerging stock markets. The regression estimates of the U.S. stock returns show mixed effects on emerging stock markets returns. Returns of 14 emerging markets have co-movement with the U.S. stock returns, while 5 markets moved in opposite direction to the U.S. market. India, Korea, Malaysia, Mexico, Nigeria, Philippine, Portugal, Thailand and Turkey returns have tendency to be affected by the U.S. stock returns positively, while Pakistan's returns are likely to be in opposite direction. Returns of Argentina, Brazil, Chile, Jordan, Taiwan, Venezuela and Zimbabwe have not influenced by the U.S. stock returns. This may be due the fact that these markets may have their own pace that may be slow than the U.S. market. In other words, these markets are isolated from the world i.e. world financial activities cannot affect these markets activities.

Table 2 presents the results of post-financial reforms in which most of the emerging markets were almost fully opened to foreign investment. The global factor (the U.S. lagged returns), after financial reforms, has dominant impact on emerging markets returns. The returns of Argentina, Brazil, Chile, Colombia, Greece, Jordan, Korea, Mexico, Portugal, Taiwan, Thailand, Turkey, Venezuela and Zimbabwe have co-movements with the U.S. returns, Malaysia returns have opposite direction, while returns of India, Nigeria, Pakistan and Philippine have no influence from the U.S. returns. The coefficients of the U.S. returns for 11 countries are statistically significant at 1 percent level, for one market at 5 percent level and for 3 markets at 10 percent level. It implies that most of the emerging markets have greater tendency towards integration, while few markets are isolated from the world market.

In sum, South East Asian markets have more tendencies to be influenced by world financial fluctuations followed by Europe/Mid-East/African and Latin American markets. It implies that South-East Asian markets were immature before financial reforms; therefore they got more influence from the world financial activities. On the other hand, Latin American markets showed more maturity than 
Table 1

Regression Results for Jan.1989 to Dec.1991 Dependent Variable: Emerging Markets’ Rates of Return

\begin{tabular}{|c|c|c|c|c|c|c|c|}
\hline Market & Constant & $\begin{array}{l}\text { U.S.Lagged } \\
\text { Return }(t-1)\end{array}$ & $\begin{array}{c}\text { Local } \\
\text { Lagged } \\
\text { Return }(t-1)\end{array}$ & $\begin{array}{l}\text { Local } \\
\text { Dividend } \\
\text { Yield } \\
\end{array}$ & $\begin{array}{l}\text { U.S. } \\
\text { Dividend } \\
\text { Yield }\end{array}$ & $\begin{array}{l}\text { U.S. Interest } \\
\text { Rate }\end{array}$ & $\mathrm{R}^{2}$ \\
\hline \multicolumn{8}{|c|}{ South-East Asian Markets } \\
\hline India & $\begin{array}{c}0.1288 \\
(2.86)^{*}\end{array}$ & $\begin{array}{l}0.388 \\
(1.60)^{* * *}\end{array}$ & $\begin{array}{c}-0.5637 \\
(3.24)^{*}\end{array}$ & $\begin{array}{c}-0.2333 \\
(5.03)^{*}\end{array}$ & & $\begin{array}{c}0.0426 \\
(5.11)^{*}\end{array}$ & 0.6627 \\
\hline Korea & $\begin{array}{l}-0.0258 \\
(1.94)^{* * * *}\end{array}$ & $\begin{array}{c}0.7019 \\
(2.62)^{*}\end{array}$ & $\begin{array}{l}-0.2161 \\
(1.35)\end{array}$ & & & & 0.2229 \\
\hline Malaysia & & $\begin{array}{c}0.6634 \\
(3.19)^{*}\end{array}$ & $\begin{array}{c}-0.1347 \\
(0.88)\end{array}$ & $\begin{array}{c}-0.0887 \\
(2.50)^{*}\end{array}$ & $\begin{array}{c}0.0572 \\
(2.45)^{*}\end{array}$ & & 0.408 \\
\hline Pakistan & $\begin{array}{c}0.1744 \\
(4.09)^{*}\end{array}$ & $\begin{array}{l}-0.3594 \\
(1.74)^{* * *}\end{array}$ & $\begin{array}{l}0.2121 \\
(1.16)\end{array}$ & $\begin{array}{c}-0.0263 \\
(3.80)^{*}\end{array}$ & & & 0.5553 \\
\hline Philippines & $\begin{array}{l}0.3427 \\
(1.52)\end{array}$ & $\begin{array}{c}0.9904 \\
(2.86)^{*}\end{array}$ & $\begin{array}{l}0.1214 \\
(0.7)\end{array}$ & & $\begin{array}{l}-0.1466 \\
(1.64)^{* * *}\end{array}$ & $\begin{array}{l}0.0201 \\
(1.06)\end{array}$ & 0.393 \\
\hline Taiwan & $\begin{array}{l}0.0971 \\
(0.2)\end{array}$ & $\begin{array}{l}0.3031 \\
(0.45)\end{array}$ & $\begin{array}{l}0.1324 \\
(0.7)\end{array}$ & $\begin{array}{l}0.0581 \\
(0.5)\end{array}$ & $\begin{array}{l}-0.0523 \\
(0.29)\end{array}$ & $\begin{array}{l}0.0063 \\
(0.18)\end{array}$ & 0.0735 \\
\hline Thailand & & $\begin{array}{c}0.849 \\
(3.07)^{*}\end{array}$ & $\begin{array}{c}0.3044 \\
(2.04)^{* *}\end{array}$ & & & & 0.3243 \\
\hline \multicolumn{8}{|c|}{ Latin American Markets } \\
\hline Argentina & $\begin{array}{l}0.4776 \\
(0.83)\end{array}$ & $\begin{array}{c}0.9934 \\
(0.909)\end{array}$ & $\begin{array}{c}-0.5542 \\
(3.33)^{*}\end{array}$ & $\begin{array}{c}-0.1083 \\
(2.85)^{*}\end{array}$ & $\begin{array}{c}-0.263 \\
(1.25)\end{array}$ & $\begin{array}{c}0.0982 \\
(1.91)^{* * *}\end{array}$ & 0.474 \\
\hline Brazil & & $\begin{array}{c}-0.531 \\
(0.56)\end{array}$ & $\begin{array}{c}-0.231 \\
(1.41)\end{array}$ & $\begin{array}{c}-0.0246 \\
(3.01)^{*}\end{array}$ & $\begin{array}{c}0.0422 \\
(2.18)^{* *}\end{array}$ & & 0.2495 \\
\hline
\end{tabular}


Table $1-$ (Continued)

\begin{tabular}{|c|c|c|c|c|c|c|c|}
\hline Market & Constant & $\begin{array}{l}\text { U.S.Lagged } \\
\text { Return }(t-1)\end{array}$ & $\begin{array}{c}\text { Local } \\
\text { Lagged } \\
\text { Return }(t-1)\end{array}$ & $\begin{array}{c}\text { Local } \\
\text { Dividend } \\
\text { Yield } \\
\end{array}$ & $\begin{array}{c}\text { U.S. } \\
\text { Dividend } \\
\text { Yield } \\
\end{array}$ & $\begin{array}{c}\text { U.S. Interest } \\
\text { Rate }\end{array}$ & $\mathrm{R}^{2}$ \\
\hline Chile & & $\begin{array}{l}0.0858 \\
(0.35)\end{array}$ & $\begin{array}{c}0.3268 \\
(1.74)^{* * *}\end{array}$ & $\begin{array}{l}-0.0104 \\
(1.36)\end{array}$ & & $\begin{array}{c}0.0128 \\
(1.60)^{* * *}\end{array}$ & 0.2615 \\
\hline Colombia & $\begin{array}{c}0.7759 \\
(8.40)^{*}\end{array}$ & $\begin{array}{l}-0.1142 \\
(1.01)\end{array}$ & $\begin{array}{l}-0.517 \\
(4.65)^{*}\end{array}$ & $\begin{array}{c}-0.0972 \\
(10.96)^{*}\end{array}$ & & $\begin{array}{l}-0.0123 \\
(1.15)\end{array}$ & 0.8608 \\
\hline Mexico & & $\begin{array}{c}0.7054 \\
(2.89)^{*}\end{array}$ & $\begin{array}{c}0.0816 \\
(0.5191)\end{array}$ & & & $\begin{array}{c}0.0046 \\
(2.38)^{* *}\end{array}$ & 0.2011 \\
\hline Venezuela & $\begin{array}{c}-0.7849 \\
(2.81)^{*}\end{array}$ & $\begin{array}{c}-0.3152 \\
(0.69)\end{array}$ & $\begin{array}{l}0.0252 \\
(0.15)\end{array}$ & $\begin{array}{c}-0.0715 \\
(2.46)^{*}\end{array}$ & $\begin{array}{c}0.2834 \\
(3.11)^{*}\end{array}$ & & 0.3928 \\
\hline \multicolumn{8}{|c|}{ Europe/Mid-East/African Markets } \\
\hline Greece & $\begin{array}{c}-0.186 \\
(1.31)\end{array}$ & $\begin{array}{c}0.9357 \\
(1.76)^{* * *}\end{array}$ & $\begin{array}{l}0.0704 \\
(0.4)\end{array}$ & & & $\begin{array}{l}0.0298 \\
(1.46)\end{array}$ & 0.1727 \\
\hline Jordan & $\begin{array}{l}0.2215 \\
(1.60)^{* * *}\end{array}$ & $\begin{array}{l}0.127 \\
(0.57)\end{array}$ & $\begin{array}{l}-0.2826 \\
(1.65)^{* * *}\end{array}$ & $\begin{array}{c}-0.0181 \\
(2.58)^{*}\end{array}$ & $\begin{array}{l}0.0608 \\
(1.23)\end{array}$ & $\begin{array}{c}-0.0464 \\
(2.46)^{*}\end{array}$ & 0.2147 \\
\hline Nigeria & & $\begin{array}{c}0.2923 \\
(1.88)^{* * *}\end{array}$ & $\begin{array}{c}0.2572 \\
(1.60)^{* * *}\end{array}$ & $\begin{array}{c}0.0022 \\
(1.92)^{* * *}\end{array}$ & & & 0.1475 \\
\hline Portugal & $\begin{array}{c}0.4651 \\
(1.95)^{* * *}\end{array}$ & $\begin{array}{l}0.5094 \\
(1.95)^{* * *}\end{array}$ & $\begin{array}{c}-0.158 \\
(0.85)\end{array}$ & $\begin{array}{l}-0.0391 \\
(1.99)^{* *}\end{array}$ & $\begin{array}{l}-0.1145 \\
(1.89)^{* * *}\end{array}$ & & 0.2859 \\
\hline Turkey & & $\begin{array}{c}1.0937 \\
(1.69)^{* * *}\end{array}$ & $\begin{array}{c}0.3778 \\
(2.37)^{* *}\end{array}$ & & & & 0.1839 \\
\hline Zimbabwe & $\begin{array}{c}-0.4856 \\
(2.93)^{*}\end{array}$ & $\begin{array}{l}-0.3029 \\
(1.2)\end{array}$ & $\begin{array}{l}-0.0107 \\
(0.05)\end{array}$ & & $\begin{array}{c}0.1082 \\
(1.93)^{* * *}\end{array}$ & $\begin{array}{l}0.0179 \\
(1.37)\end{array}$ & 0.4352 \\
\hline
\end{tabular}

*,**,*** Indicates significance at 1 percent, 5 percent, 10 percent level respectively.

t-statistics are in parenthesis.

Shaded area indicates only the U.S. returns are used instead of the U.S. lagged returns. 
Table 2

Regression Results for Jan.1992 to Dec.1998 Dependent Variable: Emerging Markets’ Rates of Return

\begin{tabular}{|c|c|c|c|c|c|c|c|}
\hline Market & Constant & $\begin{array}{l}\text { U.S.Lagged } \\
\text { Return }(t-1)\end{array}$ & $\begin{array}{c}\text { Local } \\
\text { Lagged } \\
\text { Return }(t-1)\end{array}$ & $\begin{array}{l}\text { Local } \\
\text { Dividend } \\
\text { Yield } \\
\end{array}$ & $\begin{array}{c}\text { U.S. } \\
\text { Dividend } \\
\text { Yield } \\
\end{array}$ & $\begin{array}{c}\text { U.S. Interest } \\
\text { Rate }\end{array}$ & $\mathrm{R}^{2}$ \\
\hline \multicolumn{8}{|c|}{ South-East Asian Markets } \\
\hline India & $\begin{array}{l}0.145 \\
(0.69)\end{array}$ & $\begin{array}{l}0.2704 \\
(0.81)\end{array}$ & $\begin{array}{l}0.0119 \\
(0.1)\end{array}$ & $\begin{array}{l}-0.0376 \\
(0.64)\end{array}$ & $\begin{array}{c}-0.024 \\
(0.47)\end{array}$ & $\begin{array}{l}-0.0107 \\
(0.66)\end{array}$ & 0.0259 \\
\hline Korea & $\begin{array}{c}0.254 \\
(3.49)^{*}\end{array}$ & $\begin{array}{c}0.9372 \\
(2.50)^{*}\end{array}$ & $\begin{array}{l}-0.1785 \\
(1.60)^{* * *}\end{array}$ & $\begin{array}{c}-0.0933 \\
(3.78)^{*}\end{array}$ & & $\begin{array}{l}-0.0266 \\
(1.77)^{* * *}\end{array}$ & 0.2493 \\
\hline Malaysia & $\begin{array}{c}0.5263 \\
(2.83)^{*}\end{array}$ & $\begin{array}{c}-0.9813 \\
(3.74)^{*}\end{array}$ & $\begin{array}{l}-0.0635 \\
(0.6)\end{array}$ & $\begin{array}{c}-0.0646 \\
(3.21)^{*}\end{array}$ & $\begin{array}{c}-0.0907 \\
(2.18)^{* *}\end{array}$ & $\begin{array}{l}-0.0412 \\
(1.81)^{* * *}\end{array}$ & 0.3592 \\
\hline Pakistan & $\begin{array}{c}0.3307 \\
(2.07)^{*}\end{array}$ & $\begin{array}{l}-0.1603 \\
(0.49)\end{array}$ & $\begin{array}{c}-0.0898 \\
(0.81)\end{array}$ & $\begin{array}{c}-0.0148 \\
(2.85)^{*}\end{array}$ & $\begin{array}{l}-0.0571 \\
(1.61)^{* * *}\end{array}$ & $\begin{array}{l}-0.0372 \\
(2.07)^{* *}\end{array}$ & 0.1249 \\
\hline Philippines & & $\begin{array}{c}-0.258 \\
(0.82)\end{array}$ & $\begin{array}{c}0.2266 \\
(1.81)^{* * *}\end{array}$ & $\begin{array}{l}-0.0499 \\
(1.14)\end{array}$ & $\begin{array}{l}0.0179 \\
(1.05)\end{array}$ & $\begin{array}{l}-0.0004 \\
(0.03)\end{array}$ & 0.2015 \\
\hline Taiwan & $\begin{array}{c}0.2164 \\
(2.38)^{* *}\end{array}$ & $\begin{array}{c}0.8562 \\
(2.97)^{*}\end{array}$ & $\begin{array}{l}-0.0175 \\
(0.15)\end{array}$ & $\begin{array}{l}-0.0845 \\
(2.03)^{* *}\end{array}$ & & $\begin{array}{l}-0.0327 \\
(2.39)^{* *}\end{array}$ & 0.157 \\
\hline Thailand & $\begin{array}{c}0.2384 \\
(4.62)^{*}\end{array}$ & $\begin{array}{c}1.476 \\
(4.06)^{*}\end{array}$ & $\begin{array}{c}-0.1591 \\
(1.60)^{* * *}\end{array}$ & $\begin{array}{c}-0.0505 \\
(5.29)^{*}\end{array}$ & & $\begin{array}{c}-0.0307 \\
(2.73)^{*}\end{array}$ & 0.3982 \\
\hline \multicolumn{8}{|c|}{ Latin American Markets } \\
\hline Argentina & & $\begin{array}{c}1.6736 \\
(6.03)^{*}\end{array}$ & $\begin{array}{c}0.086 \\
(0.93)\end{array}$ & & $\begin{array}{r}0.011 \\
(1.08)\end{array}$ & $\begin{array}{l}-0.0104 \\
(1.93)^{* * *}\end{array}$ & 0.3131 \\
\hline Brazil & & $\begin{array}{c}1.6917 \\
(4.82)^{*}\end{array}$ & $\begin{array}{c}0.1725 \\
(1.79)^{* * *}\end{array}$ & $\begin{array}{c}-0.0111 \\
(2.58)^{*}\end{array}$ & & & 0.2321 \\
\hline
\end{tabular}


Table 2-(Continued)

\begin{tabular}{|c|c|c|c|c|c|c|c|}
\hline Market & Constant & $\begin{array}{l}\text { U.S.Lagged } \\
\text { Return }(t-1)\end{array}$ & $\begin{array}{c}\text { Local } \\
\text { Lagged } \\
\text { Return }(t-1)\end{array}$ & $\begin{array}{l}\text { Local } \\
\text { Dividend } \\
\text { Yield } \\
\end{array}$ & $\begin{array}{l}\text { U.S. } \\
\text { Dividend } \\
\text { Yield } \\
\end{array}$ & $\begin{array}{c}\text { U.S. Interest } \\
\text { Rate }\end{array}$ & $\mathrm{R}^{2}$ \\
\hline \multirow[t]{2}{*}{ Chile } & 0.195 & 1.0823 & 0.0603 & -0.0367 & & -0.0181 & 0.3347 \\
\hline & $(3.55)^{*}$ & $(5.09)^{*}$ & $(0.6)$ & $(3.45)^{*}$ & & $(2.26)^{* *}$ & \\
\hline \multirow[t]{2}{*}{ Colombia } & 0.0917 & 0.5418 & 0.2214 & -0.0102 & & -0.0163 & 0.2121 \\
\hline & $(2.01)^{* *}$ & $(2.22)^{* *}$ & $(2.14)^{*}$ & $(1.12)$ & & $(1.66)^{* * *}$ & \\
\hline \multirow[t]{2}{*}{ Mexico } & 0.1358 & 1.6183 & 0.1888 & -0.0375 & & -0.0229 & 0.3245 \\
\hline & $(2.25)^{* *}$ & $(5.24)^{*}$ & $(1.88) * * *$ & $(1.39)$ & & $(1.91)^{* * *}$ & \\
\hline \multirow[t]{2}{*}{ Venezuela } & & 0.8424 & -0.2507 & -0.0117 & & & 0.0841 \\
\hline & & $(1.87)^{* * *}$ & $(2.25)^{* *}$ & $(1.64)^{* * *}$ & & & \\
\hline \multicolumn{8}{|c|}{ Europe/Mid-East/African Markets } \\
\hline \multirow[t]{2}{*}{ Greece } & 0.1586 & 0.3341 & -0.2515 & -0.0083 & -0.0261 & -0.0122 & 0.3772 \\
\hline & $(3.21)^{*}$ & $(1.64)^{* * *}$ & $(2.07)^{* *}$ & $(2.48)^{*}$ & $(2.59)^{*}$ & $(1.79)^{* * *}$ & \\
\hline \multirow[t]{2}{*}{ Jordan } & 0.0387 & 0.2167 & 0.0027 & 0.0014 & -0.0031 & -0.007 & 0.06 \\
\hline & -0.89 & $(1.80)^{* * *}$ & $(0.02)$ & $(0.36)$ & $(0.32)$ & (1.17) & \\
\hline \multirow[t]{2}{*}{ Nigeria } & 0.0476 & -0.0652 & -0.0642 & -0.0182 & 0.0369 & -0.004 & 0.0481 \\
\hline & $(0.27)$ & $(0.11)$ & $(0.56)$ & $(1.16)$ & $(0.75)$ & $(0.16)$ & \\
\hline \multirow[t]{2}{*}{ Portugal } & 0.0818 & 0.5843 & -0.1423 & -0.0441 & 0.0396 & -0.0075 & 0.2016 \\
\hline & $(1.28)$ & $(3.01)^{*}$ & $(1.25)$ & $(3.26)^{*}$ & $(1.83)^{* * *}$ & $(0.85)$ & \\
\hline \multirow[t]{2}{*}{ Turkey } & & 2.1044 & -0.1487 & -0.0371 & 0.0578 & & 0.179 \\
\hline & & $(3.42)^{*}$ & $(1.44)$ & $(2.23)^{* *}$ & $(2.04)^{* *}$ & & \\
\hline \multirow[t]{2}{*}{ Zimbabwe } & & 1.3318 & 0.2105 & -0.0187 & 0.0589 & -0.0172 & 0.2836 \\
\hline & & $(3.87)^{*}$ & $(2.03)^{* *}$ & $(2.31)^{* *}$ & $(2.84)^{*}$ & $(2.51)^{*}$ & \\
\hline
\end{tabular}

*,**,*** Indicates significance at 1 percent, 5 percent, 10 percent level respectively.

$t$-statistics are in parenthesis.

Shaded area indicates only the U.S. returns are used instead of the U.S. lagged returns. 
South-East Asian and Europe/Mid-East/African markets. By relaxing all the financial restrictions, South-East Asian interactions with the world markets declined, thus reflected more maturity than Latin American and Europe/Mid-East/African markets. The negative response of Malaysia, Pakistan, Philippines and Nigeria, after financial reforms, to the world market should be attractive for international investors who are looking for diversification opportunities.

Local lagged returns of emerging markets have equivalent positive and negative impact on returns of these markets. The lagged returns of 9 markets have negative impact on its returns, while 10 markets affect its returns positively. Regression coefficients of 8 markets are statistically significant, 3 out of 4 with positive sign are statistically significant at 1 percent level, while one is at 10 percent level. Similarly, 2 out of 4 with negative sign are significant at 5 percent level, while 2 at 10 percent level. The remaining 11 coefficients of lagged returns with either signs have insignificant impact on returns. These results imply that emerging market lagged returns have no significant explanatory power in determination of its returns. As stock market has an uncertain behaviour, therefore its lagged value may or may not affect its returns. This uncertain behaviour is evident from the results. Similar situations are obtained after financial reforms because most of the markets lagged returns have negative relation to their returns. This may be due to bearish trend in these stock markets or due to inefficiency of these stock markets.

Keeping in view the above discussion, we cannot draw some concrete conclusion on the basis of lagged returns about capital market segmentation. In other words, lagged returns give no information about capital markets to the potential investors.

The local instrument (emerging market dividend yield) has no predictive power to explain changes in returns for half of the emerging markets. Dividend yield of Argentina, Brazil, Colombia, India, Jordan, Malaysia, Nigeria, Pakistan, Portugal and Venezuela has inverse relation with their rate of returns. The coefficients of 8 markets are significant at 1 percent level, while 2 markets have significance at 10 percent level. The inverse relation of returns and dividend yield implies higher returns with low dividend yield.

The emerging markets dividend yields, after financial reforms, have a dominant role in the determination of its returns. The negative relation between returns and dividend yield implies that the markets with lower returns pay higher dividend yield. In other words, with the increase of dividend yield, rate of return decreases. This negative relation is significant at 1 percent level for 8 markets, at 5 percent and 10 percent level only for one market each, while other 9 markets returns have no relation with its dividend yield.

The local instruments, before financial reforms, provide no useful information to the potential investors. But in the period of financial reforms, it showed some indication to potential investors, which is also an indication of emerging markets maturity. Now investors can easily invest and have confidence in these markets. 
The global instruments (the U.S. dividend yield and interest rate) have no significant effect on emerging markets returns, which implies isolation of these markets from the world. The U.S. dividend yield has insignificant impact on most of the emerging markets returns. Only for six markets, the U.S. dividend yield has explanatory power, for two markets it is significant at 1 percent level, for one market at 5 percent level, while for 3 markets at 10 percent level.

After financial reforms, the global factors (the U.S. dividend yield and interest rate) have no influence on emerging markets returns. This may be due to the isolation of these markets from the world or they may have their own pace of adjustment to world financial activities.

The global instruments (U.S. interest rate and dividend yield) have no role to predict emerging market returns in both periods i.e. pre and post financial reforms. This evidence shows that now emerging markets are quite mature and stable. The maturity and stability of these markets can attract a lot of attention of the portfolio managers of the multi-national organisation.

It is now evident that investment restrictions in emerging markets did affect their financial markets. Although some of the emerging markets have tendency towards integration, but some of the emerging markets have also tendency towards segmentation during this period. With the opening of emerging markets to foreign investment, these financial markets gained greater tendency towards integration. In other words, after financial reforms these markets are started to have a closed link with the world-developed markets. The next section will investigate, whether financial reforms actually provided a break through for the integration of emerging markets.

\section{CHOW TEST ANALYSIS}

Chow Stability test has been applied to check the robustness of coefficients across pre-financial reforms and post-financial reforms. Chow test statistics are reported in Table 1 (see Appendix). It implies that structural change has occurred in the financial markets of Argentina, Chile, India, Korea, Mexico, Pakistan, Thailand, Turkey, and Venezuela. This structural change may be due the financial reforms started in the end of 80s. Argentina abolished all limits on foreign capital in December 1989; Chile introduced non-central bank foreign exchange market authorisation in April 1990. India took two steps in 1992, first in March 1992 when managed exchange rate abolished and the second in November when all shares on India stock markets were opened to all. Similarly, Korea's market has gone through several reforms over the period December 1989 to January 1992. These reforms include sweeping liberalisation and increased foreign ownership levels. The markets of Mexico, Pakistan, Thailand, Turkey and Venezuela have also made their stock market shares fully accessible to foreign investors. 
The markets, in which no structural change has occurred after 1991, may have gone through such change before this period. Since information of some markets about their reforms is not available, therefore some meaningful conclusion cannot be drawn.

The financial reforms during early 1990s did affect all the markets of emerging markets generally and their financial markets particularly. It is also evident that many emerging markets have gone through a structural change after these reforms.

\section{CONCLUSION}

One of the objectives of this analysis was to investigate the correlation of regression estimates of the expected returns in emerging stock markets and the U.S. stock market. The second objective was to check the effects of investment barriers on the integration/segmentation of emerging stock markets. For this purpose, using a general asset-pricing model for the pre and post-financial reforms periods performed regression analysis. Chow Stability test has been applied to check the robustness of regression coefficients across the pre-financial and post-financial reforms period.

The most important conclusion that emerges from our results is that emerging stock markets have mixed movement with the global capital market. The investment barriers did affect the integration of these markets with the global market. Before financial reforms, 73 percent emerging markets have tendency of co-movements with the world market, while 27 percent markets have movements in opposite direction. Out of 73 percent markets, only 64 percent markets returns are influenced by the world market's returns, while 36 percent markets have no response to world market. It implies that some emerging markets did integrate with the global market, but on the other hand some emerging markets have also tendency towards segmentation.

The huge capital inflow to emerging markets in early 1990s after financial reforms provided a great boost to their integration with the global market. About 78 percent emerging markets gained more tendencies to integrate with the world capital market, out of which 93 percent markets are likely to have more influence from the world market. It implies that now most of the emerging market's returns are identical to world market's returns. These results also lead to the implications of Equity Expectation Parity theorem proposed by Fisher i.e. equity returns would tend to equalise across international boundaries due to arbitrage, if there are relatively little capital control.

The local instruments (lagged returns and dividend yield) have no role in forecasting of emerging stock markets returns. This leads to uncertain behaviour of stock markets. Although local dividend yield, after financial reforms, gives some signals to potential investors, but still unable to depict all certain situation of stock market. On the other hand, global instruments are totally unable to predict emerging markets returns. These inabilities of global factors indicate the maturity and stability 
of emerging stock markets, which they have attained after financial reforms. This may be helpful to attract portfolio managers of multinationals.

The test for structural change clearly shows that most of emerging markets have gone through a structural change and this change may be due to financial reforms in these markets. This also indicates that financial reforms have provided a great boost for the stability and maturity of emerging stock markets.

As Pakistan is the leading emerging market and its returns have negative relation with global capital market and other emerging markets, therefore, policymakers should think how to attract foreign capital inflow. This can be possible if regulatory framework and structural safeguards are provided to foreign investors that characterise in developed equity markets.

Appendix Table 1

Results of Chow Stability Test

\begin{tabular}{lcccc}
\hline Markets & $F$-statistics & Probability & Log of LR & Probability \\
\hline Argentina & 7.48 & 0.00 & 54.54 & $0.00^{*}$ \\
Brazil & 1.12 & 0.35 & 4.71 & 0.32 \\
Chile & 2.68 & 0.02 & 16.69 & $0.01^{*}$ \\
Colombia & 2.35 & 0.06 & 9.71 & 0.05 \\
Greece & 0.58 & 0.65 & 2.48 & 0.65 \\
India & 4.53 & 0.0001 & 31.45 & $0.00005^{*}$ \\
Jordan & 0.41 & 0.80 & 1.74 & 0.78 \\
Korea & 2.49 & 0.04 & 12.86 & $0.02^{*}$ \\
Malaysia & 0.74 & 0.68 & 8.69 & 0.56 \\
Mexico & 2.17 & 0.05 & 13.67 & $0.03^{*}$ \\
Nigeria & 0.17 & 0.996 & 1.84 & 0.993 \\
Pakistan & 2.87 & 0.03 & 11.73 & $0.02^{*}$ \\
Philippines & 0.54 & 0.74 & 2.95 & 0.70 \\
Portugal & 0.19 & 0.967 & 1.01 & 0.961 \\
Taiwan & 0.19 & 0.90 & 0.61 & 0.89 \\
Thailand & 3.22 & 0.006 & 19.81 & $0.002^{*}$ \\
Turkey & 2.76 & 0.03 & 11.29 & $0.02^{*}$ \\
Venezuela & 3.24 & 0.014 & 13.15 & $0.011^{*}$ \\
Zimbabwe & 1.58 & 0.17 & 8.55 & 0.13 \\
\hline * Indicates significant values, i.e., there is a structural change. & &
\end{tabular}

* Indicates significant values, i.e., there is a structural change.

\section{REFERENCES}

Adler, Michael, and Bernard Dumas (1983) International Portfolio Choice and Corporation Finance: A Synthesis. Journal of Finance 38, 925-984. 
Alexander, G. J., C. S. Eun, and S. Janakiramanan (1987) Asset Pricing and Dual Listing of Foreign Capital Market: A Note. The Journal of Finance 42: 1, 151158.

Bekaert, G. (1995) Market Integration and Investment Barriers in Emerging Equity Markets. The World Bank Economic Review 9:1, 75-107.

Bekaert, G., and R. Hodrick (1992) Characterising Predictable Components in Excess Returns on Equity and Foreign Exchange Markets. Journal of Finance 47:2, 467-509.

Buckberg, E. (1995) Emerging Stock Markets and International Asset Pricing. The World Bank Economic Review 9:1, 51-74.

Campbell, J. Y. and Y. Hamao (1992) Predictable Stock Returns in the United States and Japan: A Study of Long-term Capital Integration. Journal of Finance 47: 1,43-69.

Cho, D. Chinhyung, Cheol S. Eun, and Lemm W. Senbet (1986) International Arbitrage Pricing Theory: An Empirical Investigation. Journal of Finance 41, 313-330.

Chuhan, P. (1992) Sources of Portfolio Investment in Emerging Markets. Working Paper. International Economics Department, World Bank, Washington, D. C.

Domowitz, I., J. Glen, and A. Madhavan (1997) Market Segmentation and Stock Prices: Evidence from an Emerging Market. The Journal of Finance 523 10591085.

Errunza, V., and E. Losq (1985) International Asset Pricing under Mild Segmentation: Theory and Test. The Journal of Finance 4: 1, 105-124.

Eun, C. S., and S. Janakiramanan (1986) A Model of International Asset Pricing with a Constraint on the Foreign Equity Ownership. The Journal of Finance 41:4, 897-914.

Eun, C., and S. Shim (1989) International Transmission of Stock Market Movements. Journal of Financial and Quantitative Analysis 24, 241-256.

Gultekin, M. N., N. B. Gultekin, and A. Penati (1989) Capital Controls and International Capital Market Segmentation: The Evidence from the Japanese and American Stock Market. The Journal of Finance 44:3, 849-869.

Harvey, C. R. (1995) The Risk Exposure of Emerging Equity Markets. The World Bank Economic Review 9:1, 19-50.

Hietala, P. T. (1989) Asset Pricing in Partially Segmented Markets: Evidence from the Finish Market. The Journal of Finance 44:3, 697-718.

Jorion, P., and E. Schwartz (1986) Integration vs. Segmentation in the Canadian Stock Market. The Journal of Finance 41: 3, 603-614.

Khilji, Nasir M. (1993) The Behaviour of Stock Returns in an Emerging Market: A Case Study of Pakistan. The Pakistan Development Review 32:4 593-604.

Markowitz, H. M. (1959) Portfolio Selection: Efficient Diversification of Investments. New York: John Wiley and Sons Inc. 
Shah, Salman (1992) Capital Market Development in Pakistan. In Anjum Nasim Financing Pakistan's Development in the 1990s. Lahore University of Management Sciences. Karachi: Oxford University Press.

Sharpe, W. (1964) Capital Asset Prices: A Theory of Market Equilibrium under Conditions of Risk. Journal of Finance 19:3, 425-442.

Solnik, Bruno (1974a) An Equilibrium Model of the International Capital Market. Journal of Economic Theory 8,400-424.

Stehle, Richard (1977) An Empirical Test of the Alternative Hypothesis of National and International Pricing of Risky Assets. Journal of Finance 32, 493-502.

Stulz, R. M. (1981) On the Effects of Barriers to International Investment. The Journal of Finance 36: 4, 923-934.

Tesar, Linda, and Ingrid Werner (1995) U.S. Portfolio Investment and Emerging Stock Markets. The World Bank Economic Review 9:1, 109-130.

Uppal, Jamshed Y. (1993) The Internationalisation of the Pakistani Stock Market: An Empirical Investigation. The Pakistan Development Review 32:4, 605-618.

Wheatley, Simon M. (1988) Some Tests of International Equity Integration Journal of Financial Economics 21, 177-212. 


\section{Comments}

This paper is an attempt in the area of international portfolio diversification through capital markets. This paper possibly provides guidelines to the investors for whom the restrictions are removed to own an asset in other foreign markets. The paper starts with the concept of segmentation and integration of capital markets depending on the magnitude of correlation coefficients. The segmentation arises due to government restrictions, and the markets are integrated if assets with perfectly correlated rates of return have the same price.

In this paper two approaches are mentioned to test the degree of market segmentation and integration. In first approach direct evidence of barriers and restrictions are compared with the situation where restrictions and barriers were removed. What I believe authors mentioned of comparing the coefficients of correlation between the two capital markets during pre and post-financial liberalisation periods. The second approach assumes some mean-variance efficient benchmark portfolio is observable. The expected returns are determined by the observable betas, with the benchmark return and by the observable mean benchmark return.

When I see the results in Table 1 and Table 2 which provides the regression results, I have some concern when I see the setup of the paper. For example, the empirical results presented in these tables are based on multi-factor models, which include international and domestic factors affecting and predicting the returns in any emerging market. It is important to note that the study started with looking for correlation between an emerging market and the world market (proxy with US market) during pre and post-liberalisation. But rather than providing us linear association or co-movement between two markets, the regression provides the casual relationship between the return of an emerging market and international factors like U.S. return and U.S. dividend, and domestic factors like own return and own dividend.

In segmentation and integration test in literature normally each emerging market is regressed with world price and square root of coefficient and regression coefficient provides the direction of co-movement. Then the higher value of correlation gives close co-movement of two markets. The coefficients in Tables 1 and 2 provide that what happens to return of emerging market when international and domestic factors change by one unit in respective periods. Technically it does not provide co-movement but the responsiveness of each factor on return of each emerging market.

Now some observation on these results presented in Tables 1 and 2. These models predict on time series data may arise some econometric problem. For 
example without testing for stationarity of variable may give bias results. On the model specification issues, nothing has been highlighted as in some models lag variables are used and dropped from the model or current and lag variables are used interchangeably. No justification or significance test is provided like F-test etc. Also the justification for number of lags is not given. In some cases return is used rather than excess return in case of capital asset pricing model. Nothing is mentioned about the risk-free return to calculate the excess return in each market. For example for Pakistan 6-month SBP bond rate is commonly used if the treasury bill rate is not available for the period [Nishat (2000)].

Now I would like to give some observations from the latest finance/ international finance literature, which may be more relevant for this study. For example, allowing for foreign investors in most emerging markets have resulted increased volatility as to attract foreign capital in these markets are very competitive and risky. Due to this reason, using the methodology to determine the stochastic trend to find short run and long run linkages between the two markets tests the segmentation and integration. This approach also provides the strength of linkage overtime.

In last I would like to suggest that if possible authors may time line the various financial liberalisation policies in each market to explain the extent of integration and segmentation in respective markets. Also the descriptive statistics for various indicators of each market like liquidity, size, average return etc. which provide the relative breadth and depth of these emerging markets. The study should also address about the stability of parameters across pre and post-liberalisation in these markets. For example, the study does not mention if the break points are same for each market and why?

Institute of Business Administration,

Mohammed Nishat

Karachi. 